\title{
Development and evaluation of a score to predict difficult epidural placement during labor.
}

\begin{abstract}
Abbreviated title: Difficult epidural placement during labor
\end{abstract}
Jean Guglielminotti, MD (1,4); France Mentré, MD, PhD (2,3,4); Ennoufous Bedairia, MD (1) ; Philippe Montravers, MD, PhD (1, 3); Dan Longrois, MD, PhD (1,3).

(1) Département d'Anesthésie-Réanimation, Hôpital Bichat, AP-HP, 46 rue Henri Huchard, F-75018 Paris, France

(2) Service de Biostatistique, Hôpital Bichat, AP-HP, F-75018 Paris, France

(3) Université Paris Diderot, Sorbonne Paris Cité, F-75018 Paris, France

(4) INSERM, UMR 738, F-75018 Paris, France

Correspondence: Jean Guglielminotti, Département d'Anesthésie-Réanimation, Hôpital Bichat, AP-HP, 46 rue Henri Huchard, F-75018 Paris, France

e-mail : jean.guglielminotti@bch.aphp.fr

Phone number: 33140258355

Fax number: 33140256309 


\begin{abstract}
Background and Objectives: Difficult epidural placement (DEP) during labor may be distressing for the patient and may increase the risk of dural puncture. A score predicting DEP based on the combination of individual risk factors could identify high-risk patients. Therefore, this study aimed to identify risk factors for DEP and build a prediction score.

Methods: 330 patients were prospectively included. More than one skin puncture with Tuohy needle defined DEP. Dura puncture occurrence was recorded. The population was randomly split into a training (Tra) and a validation (Val) sets. In Tra, risk factors were identified with logistic regression and used to build a score defining 3 risk-groups. Model and score discrimination was assessed with the c-index and clinical usefulness of the score with decision curves.
\end{abstract}

Results: DEP frequency was 30\% (95\%CI: 25-35). Dural puncture was more frequent in DEP patients (4\% versus $0 \%, \mathrm{p}=0.007)$. Three independent risk factors for DEP were identified: difficult interspinous space palpation (OR 6.1; 2.8-13.9), spinal deformity (OR 2.4; 1.1-5.3) and inability to flex the back (OR 3.0; 1.2-7.8). C-index of the model was $0.81(0.74-0.88)$ in Tra and $0.78(0.70-0.86)$ in Val. A 5-point score was created defining a low- (score 0), intermediate- (score 1-2) and high-risk groups (score 3-4) with predicted rates of DEP of $9.7 \%, 30.3 \%$ and $68.9 \%$, respectively. The c-index of the score was $0.79(0.72-0.86)$ in Tra and $0.76(0.69-0.84)$ in Val. Decision curves support the clinical usefulness of the score.

Conclusions: This study confirms risk factors for DEP and proposes a score predicting DEP. The score identifies high-risk patients that may benefit from an intervention to decrease DEP. This hypothesis should be evaluated in an impact study.

Key words: Analgesia, Epidural; Labor, Obstetric; Ultrasonography; Decision curve analysis; Prediction rule; Prediction model 


\section{Introduction}

Epidural analgesia during labor is commonly used worldwide with rates up to $70 \%$ in France or in the USA ${ }^{1,2}$. Multiple attempts at epidural placement with multiple needle punctures and multiple changes in needle direction may be distressing for the patient and may increase the risk of dural puncture. Dural puncture remains the most frequent complication of epidural placement during labor ${ }^{3}$. It may compromise the quality of the postpartum period and lead to claim ${ }^{4,5}$. Five risk factors for difficult neuraxial block have been reported in the literature: difficult palpation of bony landmarks, obesity, spinal deformity, inability of the patient to flex his/her back and operator's low level of experience ${ }^{6-10}$. Unfortunately, risk factors have been identified in studies based on heterogeneous populations (i.e. obstetric and non-obstetric patients) using heterogeneous techniques (i.e. spinal or epidural block and lumbar or thoracic epidural block) ${ }^{6-10}$. They may therefore not apply to epidural placement in obstetric patients. Moreover, the two studies specifically conducted in obstetric patients focused mainly on obesity and the operator's level of experience ${ }^{9,10}$. The first hypothesis tested in this study is that the 5 risk factors for difficult neuraxial block reported in the literature are also risk factors for difficult epidural placement (DEP) during labor.

Targeting interventions to patients at high-risk of DEP may decrease DEP frequency, improve patient satisfaction and reduce the risk of dural puncture. Interventions may include performing epidural placement in the sitting position that facilitates landmark location, referral to an experienced anesthesiologist to perform the procedure or the use of ultrasonographic identification of the epidural space ${ }^{11-16}$. The use of ultrasound identification of the epidural space 
when epidural puncture is expected difficult is recommended by the recent English National Institute for Health and Clinical Excellence (NICE) guidelines ${ }^{12}$. High-risk patients could be identified with a score based on the combination of individual risk factors but no score for DEP during labor is available. Determining the value of using interventions in high-risk patients identified with a score requires an impact study. Decision analysis techniques such as decision curves can estimate if a decision to use intervention in a patient is better made with or without a score ${ }^{17}$. The second hypothesis tested in this study is that a score predicting the risk of DEP is clinically useful for the decision-making process

The primary aim of this cross-sectional study was to confirm risk factors for DEP during labor and the secondary aim to build and evaluate the clinical usefulness of a score predicting the risk of DEP.

\section{Methods}

This prospective cross-sectional study was approved by the Cochin Hospital Ethics Committee, Paris, France. It was conducted in the Bichat Hospital maternity unit (a teaching hospital with 2,200 deliveries per year and $87 \%$ epidural analgesia rate). It complied with the Strengthening the Reporting of Observational Studies in Epidemiology (STROBE) statement.

\section{Study design}

Patients entering labor and requesting lumbar epidural analgesia were invited to participate by one of the investigators. Exclusion criteria were a contraindication to epidural analgesia, patient's refusal to participate or history of spinal surgery. Informed written consent was obtained from each participant. 
Investigators were 6 certified anesthesiologists and 8 residents or fellows, representing $50 \%$ of the operators performing epidural placement in our institution during the study period. Anesthesiology residents were allowed to participate in the study once they had performed 50 epidural placements and were at the 50 procedure level at the beginning of the study.

Epidural placement was performed in the sitting position as it is routine practice in our hospital. After skin anesthesia with 2\% lidocaine without epinephrine, an 18-gauge $80 \mathrm{~mm}$ Tuohy needle was inserted via the midline approach. The epidural space was identified by loss of resistance to saline. The choice of epidural space (L4-L5 or L3-L4) was left to the discretion of the attending anesthesiologist. When needle placement was not possible at the selected interspinous space, the anesthesiologist was free to choose another space. According to our local protocols, residents were not allowed to perform more than 2 skin punctures with Tuohy needle and had to ask their senior for help in that case.

\section{Definitions of DEP and dural puncture}

Epidural placement was defined difficult when Tuohy needle placement in the epidural space required more than one skin puncture, regardless of the number of needle redirections. This definition of DEP was based on a survey of the literature about difficult neuraxial block ${ }^{6-8,10}$. In this survey, the most frequently used definition was the number of skin puncture and the most frequently used threshold defining difficulty was more than one puncture.

Dural puncture was self-reported by each investigator. It was defined as either reflux of cerebrospinal fluid in the Tuohy needle or epidural catheter or motor blockade after a test dose of $2 \mathrm{~mL} \mathrm{2 \%} \mathrm{lidocaine} \mathrm{without} \mathrm{epinephrine.} \mathrm{Motor} \mathrm{blockade} \mathrm{after} \mathrm{the} \mathrm{test} \mathrm{dose} \mathrm{was} \mathrm{defined} \mathrm{as} \mathrm{a}$ grade $\geq 2$ on the 4 -grade Bromage score with grade 1 corresponding to free movement of legs and feet and grade 4 to inability to move legs or feet. 


\section{Risk factors for DEP}

Five risk factors for DEP were selected from a survey of the literature about difficult neuraxial block ${ }^{18}$.

Palpation of interspinous spaces was classified as good when easily palpable, poor when hardly palpable and nil when impalpable ${ }^{6,7,9,10}$. Interspinous space palpation was defined difficult for palpation scores of poor or nil.

Obesity was assessed by body mass index (BMI) at term (i.e. the ratio of the weight in $\mathrm{kg}$ to the square of the height in $\mathrm{m}$ ). Since no BMI cut-off value has been defined for obesity during pregnancy and as dichotomization of a continuous variable in multivariable analysis can lead to loss of information, BMI was expressed as a continuous variable ${ }^{19}$.

Spinal deformity was defined as deviation from the midline of the visible or palpated spinal processes, as previously reported ${ }^{6,7,9,10}$.

The ability of the patient to flex her spine was observed by standing at the patient's side and asking her to flex her back to a maximum ${ }^{7,9}$. The curvature of the skin was recorded as either convex, straight, or concave. Inability to flex the spine was considered to be present when the patient presented a straight or concave curve.

Operator level of experience was based on the number of epidural placements performed by each investigator before participating in the study. An investigator was defined proficient when he/she had performed more than 100 procedures before participating in the study. The cutoff of 100 was based on the study by Konrad et al. that demonstrated that it was the number of cases necessary on the learning curve to obtain an at least $80 \%$ successful placement rate ${ }^{13}$.

\section{Outcome assessment}


The operators performing epidural placement were unblinded. They both assessed risk factors before epidural placement and reported the number of skin punctures with Tuohy needle after epidural placement on the case report form. They were instructed before participating in the study to report patient's risk factors on the case report form before performing epidural placement.

\section{Statistical analysis}

Statistical analysis used R software, version 2.14.1 ( $\mathrm{R}$ Foundation for Statistical Computing, Vienna, Austria) and the results were expressed as number of patients (\%) or mean \pm one standard deviation. When appropriate, $95 \%$ confidence intervals $(95 \% \mathrm{CI})$ were calculated.

The frequency of dural puncture was compared in patients with and without DEP with the Fisher's exact test.

The population was randomly split into two equal size sets: a training set used for model building and a validation set. The randomization was made with the function sample of the statistical software R. Firstly, 165 lines corresponding to 165 patients of the database containing 330 patients were sampled at random and without replacement to constitute the training set. Secondly, the remaining 165 patients not sampled were defined as the validation set. The five candidate variables were compared between patients with and without DEP in the training set using Wilcoxon test for continuous variables and Fisher's exact test for discrete variables. The odds ratio of DEP for each variable was calculated with univariate logistic regression. The five variables were then entered into a logistic regression analysis with backward selection to obtain a prediction model. Interactions between final variables in the prediction model were tested. The final model was evaluated for discrimination with the c-statistic. Discrimination refers to the 
model ability to discriminate a patient with DEP from a patient without DEP. The model developed in the training set was applied to the validation set without re-estimating the coefficients and evaluated for discrimination with the c-index.

Our original plan was to include at least 350 patients. Based on a prevalence of DEP of $30 \%$, 105 events were expected with half of the events in the training set and half in the validation set ${ }^{6}$, ${ }^{7}$. Since 5 candidate variables were tested in the training set, it would lead to a ratio of the number of events to the number of candidate variables greater than 10 on multivariable analysis.

A score to predict DEP was developed from the regression coefficients of the multivariable logistic regression model of the training set. Logistic regression coefficient for the 3 significant risk factors (difficult palpation of the interspinous space, spine abnormality and inability to flex the back) was rounded to the nearest integer. This integer defined the number of points attributed to the risk factor. The score was the sum of the points corresponding to each variable. Three risk groups were defined in the training set by cutoff values of the score according to sample sizes with $1 / 3$ of the training population in each risk-group. The odds ratio of DEP for the 3 riskgroups was calculated with univariate logistic regression. Discrimination of the score divided into three risk-groups in the training set was assessed with the c-statistic and calibration with a calibration plot. Calibration refers to the agreement between the predicted probability of DEP in each risk group and the observed probability of DEP in each risk group. The agreement is obtained when the predicted probability in a given group is comprised within the $95 \%$ confidence interval of the observed probability in this group. In the validation set, discrimination and calibration of the score divided into three risk-groups was also assessed with the c-statistic and a calibration plot. 
Evaluation of a strategy to use an intervention to avoid DEP based on the 3 risk groups defined by the score was made with decision curves in the training and in the validation sets. Decision curve is a graphic display of the relationship between the net benefit of the 3 risk groups defined by the score and the threshold probability for intervention ${ }^{17,20}$. In the present study, the threshold probability for intervention was the probability of DEP for a given patient in whom the anesthesiologist would use an intervention to avoid this event. A net benefit is calculated for each threshold probability. The net benefit is the probability of true-positive results minus the probability of false-positive results with the latter weighted by the odds at the threshold. Interpretation of decision curve analysis is based on comparison of the net benefit of the 3 risk groups defined by the score with the net benefit of an "intervention in all patients" strategy (all patients have DEP) and with the net benefit of an "intervention in no patient" strategy (no patient has DEP). When the net benefit of the 3 risk groups defined by the score is higher than the benefit of these two strategies, the decision to use an intervention should be based on the risk groups rather than on the anesthesiologist's opinion. Decision curve analysis allows determination of the range of threshold probabilities over which the 3 risk groups defined by the score is clinically useful ${ }^{17}$. The decision curve analysis was performed with the DCA package downloaded on www.decisioncurveanalysis.org ${ }^{21}$.

\section{Results}

Three hundred thirty patients were included in the study. The study flowchart is presented in Figure 1 and patient characteristics are shown in Table 1.

\section{Dural puncture}


The dural puncture frequency was significantly higher in patients with DEP than in patients without DEP: 4/98 (4.0\%;95\%CI: $1.1-10.1)$ versus 0/232 (0\%;0.3-3.1), respectively $(\mathrm{p}=$ 0.007).

\section{Univariate and multivariate analysis}

Prevalence of DEP was $32 \%(25-40)$ in the training set. Distribution of the 5 candidate risk factors in this set is presented in Table 1.

In the training set, the candidate risk factors were significantly different between patients with and without DEP except for the operator's level of experience (Table 2). Multivariate analysis identified three risk factors for DEP (Table 2): difficult palpation of the interspinous space, spine abnormality and inability to flex the back. No significant interaction was observed between the three final variables. The c-index of this model was $0.81(0.74-0.88)$.

The prevalence of DEP was $27 \%$ (20-35) in the validation set. The distribution of the 5 candidate risk factors in this set is presented in Table 1 . The c-index of the model built in the training set when applied to the validation set was $0.78(0.70-0.86)$.

\section{Score to predict DEP and risk groups}

The points based on the logistic regression coefficients for the presence of each of the 3 risk factors in the training set are presented in Table 2: 2 points were attributed for the presence of "difficult palpation of the interspinous space" and 1 point for the presence of "spine abnormality" or "inability to flex the back". The score was calculated by adding each component and ranged from 0 to 4 with a median value of 1 in the training set.

In the training set, 3 groups were defined: a low- (score of $0 ; 62$ patients or $37 \%$ ), an intermediate- (score of $1-2 ; 61$ patients or 37\%), and a high-risk group (score of 3-4; 42 patients 
or 26\%). Predicted probabilities of DEP were 9.7\%, 30.3\%, and 68.9\%, respectively (Figure 2). Compared with the low-risk group, odds ratio related to DEP in the training set was 3.6 (1.410.7) for the intermediate-risk group and 23.3 (8.5-74.3) for the high-risk group. The c-index of the score divided into 3 risk-groups was $0.79(0.72-0.86)$ and its calibration is shown in Figure 2.

The 3 risk groups based on the score built in the training set were evaluated in the validation set. In the validation set, predicted probabilities of DEP in the low- (78 patients or $47 \%$ ), intermediate- (56 patients or 34\%), and high-risk groups (31 patients or 19\%) were 9\%, $27.3 \%$, and $63.1 \%$ respectively (Figure 2). The c-index in the validation set was good with a value of $0.76(0.69-0.84)$. The good calibration of the score was attested by the fact that predicted probability in each group was within the $95 \%$ confidence interval of observed probability of DEP (Figure 2).

\section{Decision curve analysis}

Evaluation of a strategy to use an intervention to avoid DEP based on the 3 risk groups defined by the score was made with decision curves in the training and in the validation sets (Figure 3). In the training set, for a threshold probability between 10 and $72 \%$, the 3 risk groups defined by the score had a higher net benefit than the two extreme strategies. If a clinician would consider an intervention when the risk of DEP was $10 \%$ or higher, then this decision would be optimally guided by the 3 risk groups defined by the score rather than by clinical opinion. On the contrary, if a clinician would consider an intervention when the risk of DEP was $72 \%$ or higher, then this decision would be optimally guided without the risk groups. In the validation set, the 3 risk groups defined by the score had a higher net benefit than the two extreme strategies for a threshold probability between 10 and $61 \%$. 


\section{Discussion}

Avoiding DEP during labor is an important issue considering the high number of procedures performed annually, its prevalence (30\% in the current study) and the high frequency of dural puncture in DEP patients ( $4 \%$ in the current study).

\section{Multivariable analysis of risk factors for DEP}

Although intuitive, difficult bony landmark palpation, obesity, spinal deformity, poor back flexion and operator's low level of experience have all been identified as risk factors for difficult neuraxial block ${ }^{6-9}$. Unfortunately, the above-mentioned studies included heterogeneous populations and techniques that may preclude their applicability to epidural placement during labor. Moreover, they did not always comply with current guidelines for the construction and validation of a prediction model ${ }^{22}$. As in previous studies, we found that difficult bony landmark palpation, spinal deformity and poor back flexion were independent risk factors for DEP. The result of the multivariable analysis was not modified when defining DEP as Tuohy needle placement in the epidural space requiring more than two skin punctures. Difficult bony landmark palpation was associated with the highest risk as evidenced by an odds ratio twice the one of spinal deformity or poor back flexion. Like Ellinas et al., we did not demonstrate any effect of obesity, probably because of the association between obesity and difficult bony landmark palpation or poor back flexion ${ }^{9,11}$. The lack of statistical association between the operator's level of experience and DEP may be explained by the fact that investigators in our study were either seniors or experienced residents. The results of our study may therefore not apply to institutions with novice obstetric anesthesia residents. 
The multivariable prediction model had a good discriminating ability with a c-statistic of 0.81. However, the real performance of a prediction model is only ascertained when applied to a population different from the population used to construct the model (external validation) ${ }^{18}$. Internal validation with random splitting in a training and validation sets is a robust method to assess the performance of the model if it had to be applied to a different population. In the training set, the c-statistic was still 0.77 with a difference of the c-statistic between the training and validation sets less than 0.05 .

\section{Development of a score to predict DEP and creation of risk groups}

A method to assess individual outcome is to use a simple score combining risk factors for DEP according to their own predictive strength. The score enabled 3 risk groups to be defined stratifying the risk of DEP into low (score 0), intermediate (score 1-2) or high (score 3-4). The good discrimination of the score in the training and validation sets (c-statistic $=0.79$ and 0.76 , respectively) and its good calibration (Figure 2) show its robustness. It is an argument to support its application in clinical practice.

Interventions to avoid DEP could include performing epidural placement in the sitting position that facilitates landmark location or using ultrasonographic identification of the epidural space ${ }^{11-16,23}$. The use of ultrasound identification of the epidural space when epidural puncture is expected to be difficult is recommended by the recent NICE guidance ${ }^{12}$. Unfortunately, the guidance did not indicate when DEP is expected. Two studies conducted in obstetric patients, with one study focusing on patients with expected difficult epidural placement, reported that ultrasonography reduces the number of needle punctures required for epidural placement ${ }^{15,16}$. They also reported that ultrasonography may improve analgesia quality and parturient satisfaction. The study by Chin et al, demonstrating that ultrasonography reduced the number of 
needle puncture for spinal anesthesia in orthopedic patients with difficult surface anatomy landmarks, supports this view ${ }^{14}$. The possibility to plan interventions in order to avoid DEP highlights the importance of routinely and carefully identifying risk factors during the preanesthetic assessment performed during pregnancy or on admission to the maternity unit.

The choice of the risk group that should benefit from an intervention to avoid DEP should take into account the predicted risk of difficult epidural. In our study, this risk was less than $10 \%$ in the low-risk group (score 0), more than $25 \%$ in the intermediate-risk group (score 1-2) and more than $60 \%$ in the high-risk group (score 3-4). On this basis, we suggest that interventions should be proposed to the high-risk group and would concern $22 \%$ of the whole population we studied. A score greater than 3 that defines the high-risk group corresponds to a patient with difficult interspinous space palpation and spine abnormality, or with difficult interspinous space palpation and inability to flex the back, or with the 3 risk factors. This suggestion should be confirmed by an impact study to determine whether targeting interventions to the high-risk group

is better than usual care in decreasing the frequency of DEP and its related complications ${ }^{22,24}$. In our opinion, the impact study should investigate the benefit of ultrasonography in the high-risk group (i.e. score 3-4). Anyway, the decision curve of the 3 risk groups defined by the score suggests that the benefit of using this strategy would be superior to the one of not using it.

\section{Limitations of the study}

The definition of DEP used in this study was based on a parameter (i.e. the number of skin punctures) that was objective, easily quantifiable and that did not involve observer interpretation. It may not reflect the subjective clinical experience of senior obstetric anesthesiologists of difficult epidural placement. However, no universally accepted definition of DEP is available at the present time and our definition is in line with the one used in previous 
studies ${ }^{6-8,10}$. Moreover, a significantly higher dural puncture frequency was observed in patients with DEP suggesting that, in addition to the technical difficulty for the anesthesiologist, this definition corresponded to a poorer outcome for the parturient.

The study could have been designed as a blinded one, with an operator performing epidural placement and an independent observer recording the data. However, the $24 \mathrm{~h} / 7 \mathrm{~d}$ nature of obstetric anesthesia practice and of patients inclusion made the availability of an independent observer a practical issue.

\section{Conclusion}

In conclusion, we have confirmed risk factors for DEP during labor and proposed a score predicting DEP. The score identifies high-risk patients that may benefit from an intervention to decrease DEP. This hypothesis should be evaluated in an impact study.

\section{References}

1 Ducloy-Bouthors AS, Prunet C, Tourres J, Chassard D, Benhamou D, Blondel B. Medical care organization in analgesia, anaesthesia and intensive care in maternity units: Results from the National Perinatal Surveys in 2003 and 2010. Ann Fr Anesth Reanim 2012 (In Press).

2 Bucklin BA, Hawkins JL, Anderson JR, Ullrich FA. Obstetric anesthesia workforce survey: twenty-year update. Anesthesiology 2005; 103: 645-53

3 Cheesman K, Brady JE, Flood P, Li G. Epidemiology of anesthesia-related complications in labor and delivery, New York State, 2002-2005. Anesth Analg 2009; 109: 1174-81

4 Webb CA, Weyker PD, Zhang L, et al. Unintentional dural puncture with a tuohy needle increases risk of chronic headache. Anesth Analg 2012; 115: $124-32$

5 Davies JM, Posner KL, Lee LA, Cheney FW, Domino KB. Liability associated with obstetric anesthesia: a closed claims analysis. Anesthesiology 2009; 110: 131-9

6 Sprung J, Bourke DL, Grass J, et al. Predicting the difficult neuraxial block: a prospective study. Anesth Analg 1999; 89: 384-9 
7 de Filho GR, Gomes HP, da Fonseca MH, Hoffman JC, Pederneiras SG, Garcia JH. Predictors of successful neuraxial block: a prospective study. Eur J Anaesthesiol 2002; 19: 447-51

8 Atallah MM, Demian AD, Shorrab AA. Development of a difficulty score for spinal anaesthesia. Br J Anaesth 2004; 92: 354-60

9 Ellinas EH, Eastwood DC, Patel SN, Maitra-D'Cruze AM, Ebert TJ. The effect of obesity on neuraxial technique difficulty in pregnant patients: a prospective, observational study. Anesth Analg 2009; 109: 1225-31

10 Faitot V, Ourchane R, Dahmani S, et al. An observational study of factors leading to difficulty in resident anaesthesiologists identifying the epidural space in obstetric patients. Int J Obstet Anesth 2011; 20: 124-7

11 Ellinas EH. Focused review: labor analgesia for the obese parturient. Anesth Analg 2012; 115: 899-903

12 NICE. Ultrasound-guided catheterisation of the epidural space: guidance. http://wwwniceorguk/Guidance/IPG249/Guidance/pdf/English 2008

13 Konrad C, Schupfer G, Wietlisbach M, Gerber H. Learning manual skills in anesthesiology: Is there a recommended number of cases for anesthetic procedures? Anesth Analg 1998; 86: 635-9

14 Chin KJ, Perlas A, Chan V, Brown-Shreves D, Koshkin A, Vaishnav V. Ultrasound imaging facilitates spinal anesthesia in adults with difficult surface anatomic landmarks. Anesthesiology 2011; 115: 94-101

15 Grau T, Leipold RW, Conradi R, Martin E, Motsch J. Efficacy of ultrasound imaging in obstetric epidural anesthesia. J Clin Anesth 2002; 14: 169-75

16 Grau T, Leipold RW, Conradi R, Martin E. Ultrasound control for presumed difficult epidural puncture. Acta Anaesthesiol Scand 2001; 45: 766-71

17 Vickers AJ, Elkin EB. Decision curve analysis: a novel method for evaluating prediction models. Medical Decision Making 2006; 26: 565-74

18 Steyerberg EW, ed. Clinical prediction models: a practical approach to development, validation, and updating. New-York: Springer, 2010

19 Altman DG, Royston P. The cost of dichotomising continuous variables. British Medical Journal 2006; 332: 1080

20 Vickers AJ. Decision analysis for the evaluation of diagnostic tests, prediction models and molecular markers. Am Stat 2008; 62: 314-20

21 Vickers AJ, Cronin AM, Elkin EB, Gonen M. Extensions to decision curve analysis, a novel method for evaluating diagnostic tests, prediction models and molecular markers. BMC Med Inform Decis Mak 2008; 8: 53

22 Moons KG, Royston P, Vergouwe Y, Grobbee DE, Altman DG. Prognosis and prognostic research: what, why, and how? British Medical Journal 2009; 338: b375

23 Hayter MA, Friedman Z, Katznelson R, Hanlon JG, Borges B, Naik VN. Effect of sleep deprivation on labour epidural catheter placement. Br J Anaesth 2010; 104: 61927

24 Reilly BM, Evans AT. Translating clinical research into clinical practice: impact of using prediction rules to make decisions. Ann Intern Med 2006; 144: $201-9$ 
Table 1: Patient characteristics, frequency and risk factors for difficult epidural placement in the whole population, in the training and in the validation sets.

\begin{tabular}{|l|c|c|c|}
\hline & Whole population & Training set & Validation set \\
& $(\mathbf{n = 3 3 0 )}$ & $\mathbf{( n = 1 6 5 )}$ & $30 \pm 6$ \\
\hline Age (years) & $31 \pm 6$ & $1.8 \pm 1.2$ & $1.7 \pm 1.0$ \\
\hline Parity & $1.8 \pm 1.1$ & $4 \pm 2$ & $5 \pm 2$ \\
\hline Cervical dilation at the time of epidural (cm) & $4 \pm 2$ & $5 \pm 1$ & $45(27 \%)$ \\
\hline Epidural space depth (cm) & $5 \pm 1$ & $53(32 \%)$ & $50(30 \%)$ \\
\hline Difficult epidural placement & $98(30 \%)$ & $74(45 \%)$ & $27.4 \pm 4.0$ \\
\hline Difficult interspinous space palpation & $124(38 \%)$ & $28.0 \pm 4.6$ & $45(27 \%)$ \\
\hline BMI at term (kg/m $)$ & $27.7 \pm 4.3$ & $57(34 \%)$ & $29(18 \%)$ \\
\hline Spine abnormality & $102(31 \%)$ & $33(20 \%)$ & $104(63 \%)$ \\
\hline Inability to flex the back & $62(19 \%)$ & $111(67 \%)$ & \\
\hline Experience < 100 placements & $215(65 \%)$ & & \\
\hline
\end{tabular}

Results are expressed as mean \pm one standard deviation or number of patients (\%). BMI: body mass index 
Table 2: Univariate analysis, multivariate analysis and points for the presence of risk factors for difficult epidural placement (DEP) in the training set.

\begin{tabular}{|c|c|c|c|c|c|c|c|c|}
\hline & \multicolumn{4}{|c|}{ Univariate analysis } & \multicolumn{4}{|c|}{ Multivariate analysis } \\
\hline & $\begin{array}{l}\text { No DEP } \\
(n=112)\end{array}$ & $\begin{array}{c}\text { DEP } \\
(n=53)\end{array}$ & $\begin{array}{c}\text { OR } \\
(95 \% \mathrm{CI})\end{array}$ & p value & $\begin{array}{c}\text { OR } \\
(95 \% \mathrm{CI})\end{array}$ & p value & $\begin{array}{l}\text { Regression } \\
\text { coefficients }\end{array}$ & $\begin{array}{c}\text { Points } \\
\text { for the score }(*)\end{array}$ \\
\hline $\begin{array}{l}\text { Difficult interspinous } \\
\text { space palpation }\end{array}$ & $33(29 \%)$ & $41(77 \%)$ & $8.2(3.9-18.1)$ & $<0.0001$ & $6.1(2.8-13.9)$ & $<0.0001$ & 1.81 & 2 \\
\hline BMI at term $\left(\mathrm{kg} / \mathrm{m}^{2}\right)$ & $27.3 \pm 4.6$ & $29.5 \pm 4.3$ & $1.1(1.0-1.2)$ & 0.0018 & - & - & - & - \\
\hline Spine abnormality & $27(24 \%)$ & $30(57 \%)$ & $4.1(2.1-8.3)$ & $<0.0001$ & $2.4(1.1-5.3)$ & 0.03 & 0.87 & 1 \\
\hline Inability to flex the back & $13(12 \%)$ & $20(38 \%)$ & $4.6(2.1-10.5)$ & 0.0003 & $3.0(1.2-7.8)$ & 0.02 & 1.11 & 1 \\
\hline Experience $<100$ placements & $78(70 \%)$ & $33(63 \%)$ & $0.7(0.4-1.4)$ & 0.37 & - & - & - & - \\
\hline
\end{tabular}

Results are presented as mean ( \pm 1 SD) or number of patients (\%). OR: odds ratio, 95\%CI: 95\% confidence interval and BMI: body mass index.

(*) Logistic regression coefficient for each risk factor was rounded to the nearest integer. 
Figure 1: Study flowchart.

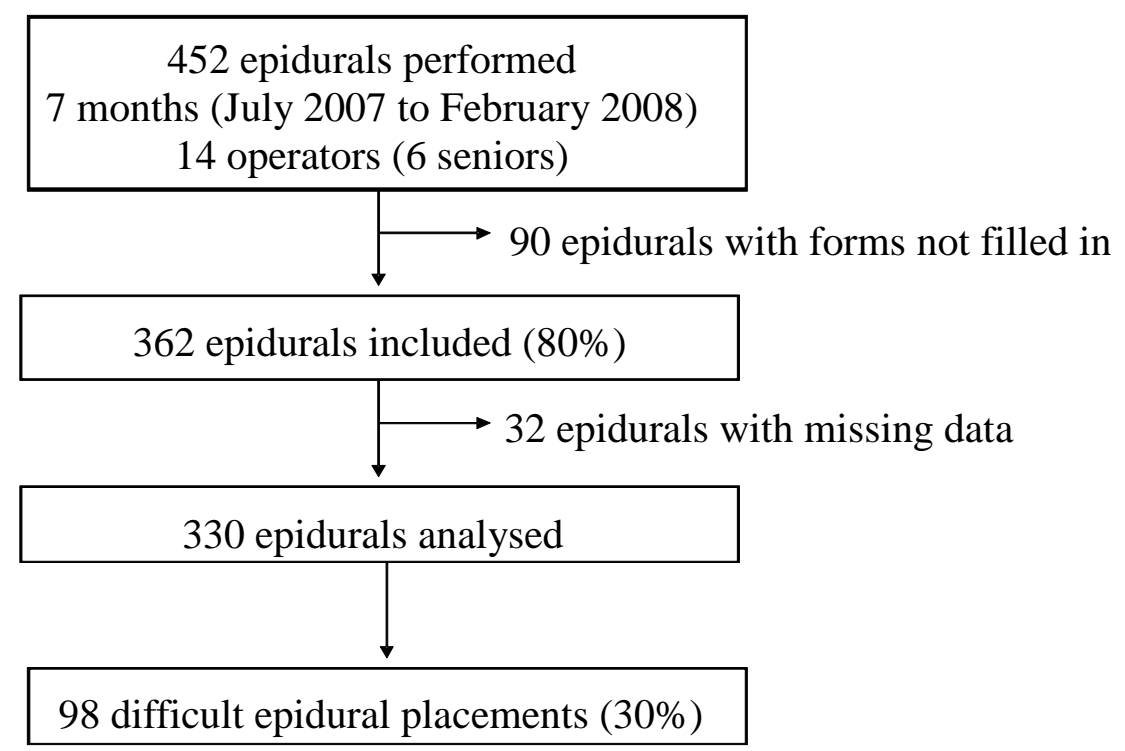


Figure 2: Assessment of the calibration of the score in the training and validation sets: predicted probabilities and observed probabilities with $95 \%$ confidence interval of difficult epidural placement according to the 3 risk groups defined by the score. $\mathrm{n}$ refers to the number of patients in each risk group.

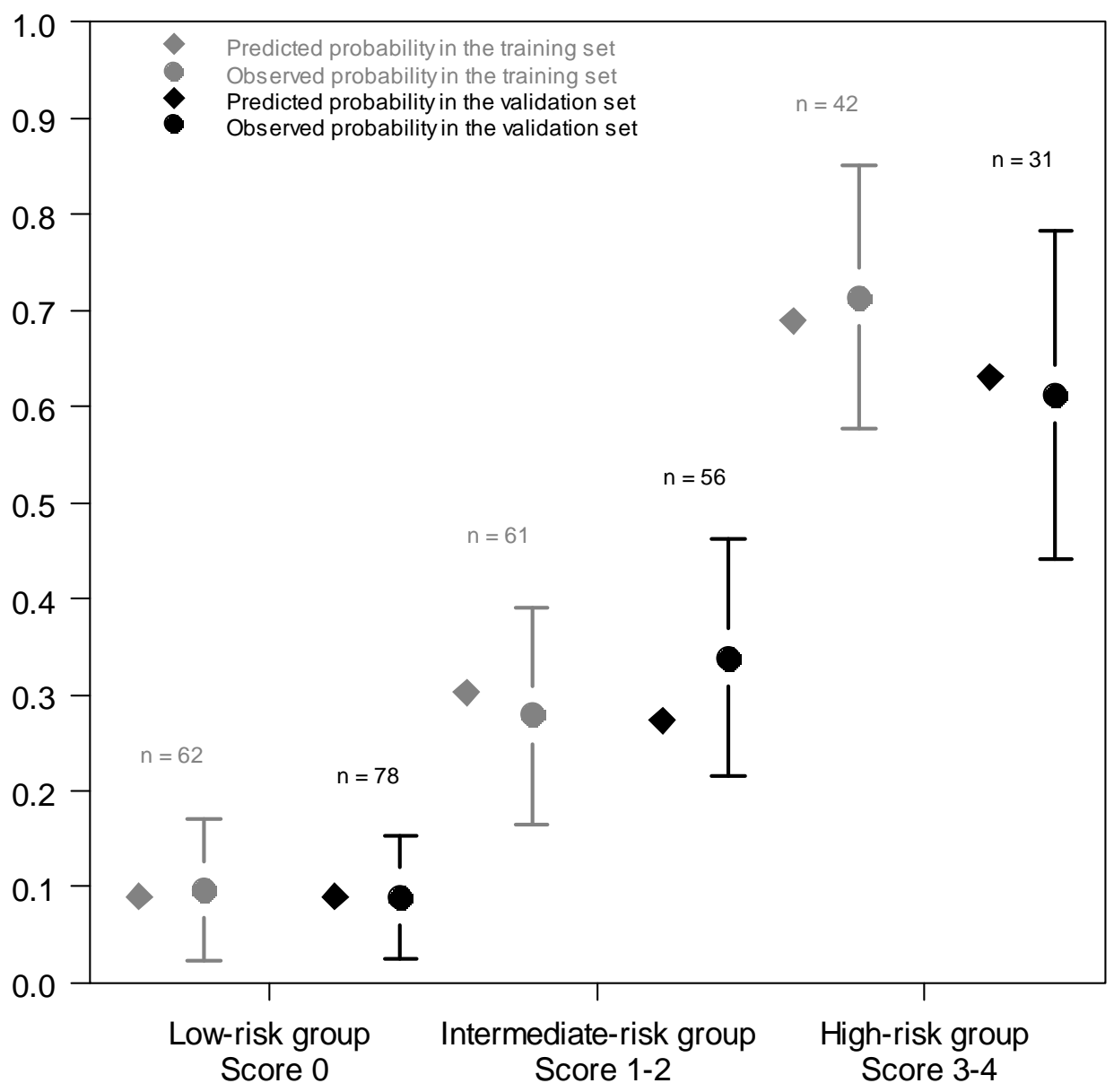


Figure 3: Decision curves illustrating the relationship between net benefit of an intervention based of the 3 risk groups defined by the score and threshold probability for intervention in the training (left panel) and validation (right panel) sets. The threshold probability for intervention is the probability of difficult epidural placement at which an anesthesiologist would use an intervention such as lumbar spine ultrasonography to avoid this event. When the net benefit of the "intervention according to risk groups" is superior to the net benefit of the two extreme strategies, the decision to intervene should be guided by the 3 risk groups defined by the score rather than by the anesthesiologist's personal judgment.
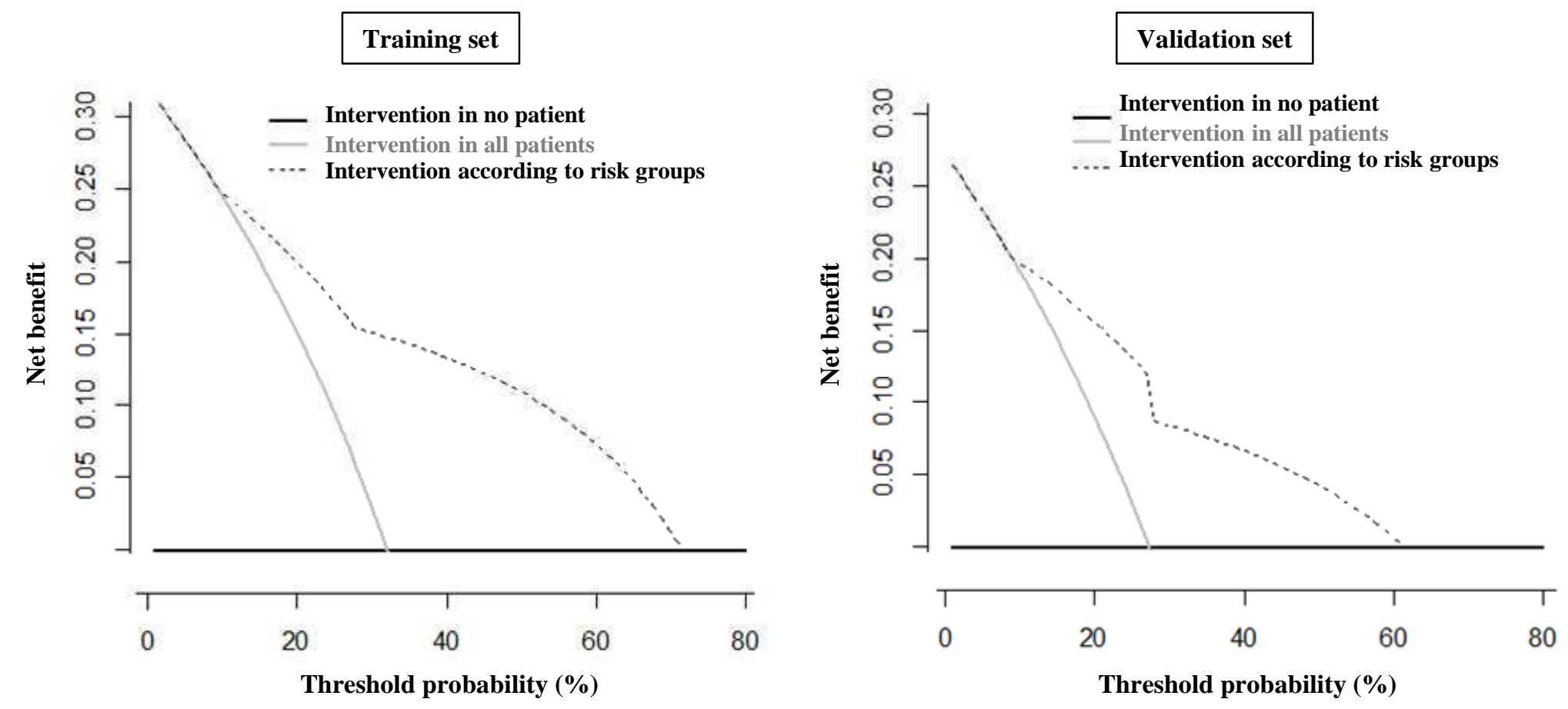